\title{
SMT: Split and Merge Tractography for DT-MRI
}

\author{
Uğur Bozkaya and Burak Acar \\ Boğaziçi University, Electrical \& Electronics Eng. Dept., VAVlab, İstanbul, Turkey \\ acarbu@boun.edu.tr, burak.acar@ieee.org \\ www. vavlab.ee.boun.edu.tr
}

\begin{abstract}
Diffusion tensor magnetic resonance imaging (DT-MRI) based fiber tractography aims at reconstruction of the fiber network of brain. Most commonly employed techniques for fiber tractography are based on the numerical integration of the principal diffusion directions. Although these approaches generate intuitive and easy to interpret results, they are prone to cumulative errors and mostly discard the stochastic nature of DT-MRI data. The proposed Split \& Merge Tractography (SMT) technique aims at overcoming the drawbacks of fiber tractography by incorporating it with Markov Chain Monte Carlo techniques. SMT is based on clustering diversely distributed short fiber tracts based on their inter-connectivity. SMT also provides real-time interaction to adjust a user defined confidence level for clustering.
\end{abstract}

\section{Introduction}

Diffusion Tensor Magnetic Resonance Imaging (DT-MRI) is the unique modality that allows in-vivo imaging of the nervous network in brain. The data is a symmetric, positive semi-definite second-order tensor field that is a second order approximation of the physical diffusion process locally. The principal eigenvectors of the tensors are shown to be aligned with the underlying fibers in regions of anisotropic diffusion. However, the DT-MRI data is derived from a set of Diffusion Weighted Magnetic Resonance Imaging (DWI) data. DWI data is acquired using diffusion weighting gradient magnetic fields, $G$, in addition to the constant field, $B_{0}$. $G$ causes the MR signal to attenuate due to the diffusion along this magnetic field gradient [1,2,3].

It is of utmost importance to understand what the DT-MRI data represents in order to develop adequate analysis and visualization methods. DT-MRI represents a macro view of the diffusion process within a finite voxel volume as observed by DWI. Neither the accuracy of the second order approximation, nor its spatial resolution is adequate to represent individual fibers.

A novel approach to increase the accuracy of this approximation by using higher order tensors was proposed by Liu et al. 4]. The so called Generalized Diffusion Tensor Imaging (GDTI) has not been put in practice and will not be discussed here. The two major approaches to DT-MRI analysis and visualization are Fiber Tractography and Connectivity Mapping. The former approach solely

N. Ayache, S. Ourselin, A. Maeder (Eds.): MICCAI 2007, Part II, LNCS 4792, pp. 153 160, 2007.

(C) Springer-Verlag Berlin Heidelberg 2007 
relies on the numerical integration of the principal diffusion direction (PDD, the major eigenvector of the diffusion tensor) and attempts to reconstruct the fiber that passes through a given point 5 . The most popular method is the 4th order Runge-Kutta [6. These approaches are prone to cumulative errors and most of them oversee the stochastic nature of the underlying data 7,8 . The latter approach attempts to utilize the true nature of the DT-MRI data, i.e. the second order approximation of the physical diffusion process, by estimating a connectivity map. They consider each and every possible connection between neighbouring voxels with weights set by the dataset. Several approaches in this group are based on Monte-Carlo simulations of the random walk model 9, 10, 11. Lenglet et al., on the other hand, recasted the connectivity problem to the Riemannian differential geometry framework where they defined their local metric tensor using the DTI data and solved for geodesics [12.

The most important point that differentiates these two approaches is their response at problematic regions such as crossing, kissing and branching fibers. The tractography methods either pretend to follow a single fiber by choosing a direction to proceed at such points or stop tracking. These methods do not allow for a user interface to set a confidence level despite the nature of the data. The connectivity mapping methods, on the other hand, lets the results be interpreted with respect to some confidence definition. They also allow for branching. Although single fibers do not branch, fiber bundles do and this makes branching while tracking a necessity due to the low spatial resolution of DT-MRI data. Thus, the connectivity mapping is a more direct way of communicating the stochastic and structural information embedded in the data than conventional fiber tractography. Yet the computational cost of connectivity mapping is high and their interpretation is not straight forward.

The Split \& Merge Tractography (SMT) method proposes a compromise between tractography, which greatly disregards the stochastic nature of the data and accumulates error, and connectivity mapping, which is computationally costly and hard to interpret. SMT is based on clustering short fiber tracts using a Monte Carlo Markov Chain (MCMC) approach. Using short tracts prevents error accumulation, while the MCMC provides a stochastic framework in which we can define a confidence level for the clusters, allowing the user to investigate the data in detail.

\section{Method}

Split \& Merge Tractography (SMT) 13, 14 is a MCMC technique that is used to estimate the unknown distribution of fiber tracts. However, unlike previously proposed methods that exploit the stochastic nature of DT-MRI data for fiber tractography [15, 16, the output of SMT is not the full tracts but rather clusters of short tracts. The underlying rationale behind this is to avoid the error accumulation.

The short tracts are computed by the numerical integration of the PDD field using the 4 th order Runge-Kutta method [6]. SMT avoids such error accumulation 


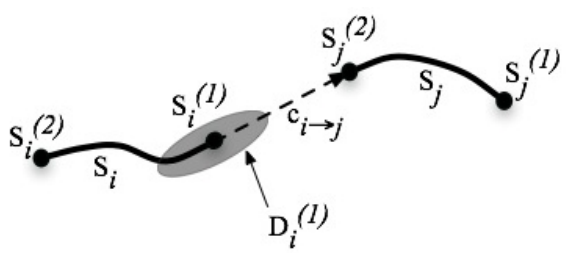

Fig. 1. A bridge is built from the current short tract $S_{i}$ (initially a seed tract) to $S_{j}$, which is selected based on the Gaussian PDF described by $D_{i}^{(1)}$

in PDD tracking by using short tracts. PDD tracking is started from each voxel unless that voxel is on a previously computed short tract. The maximum length of the short tracts is set to $2.8 \mathrm{~mm}$, the tracking is terminated when the Fractional Anisotropy is below 0.25 or the curvature exceeds $20^{\circ}$ per step. This is the splitting step where the whole brain is populated by short tracts.

The merging step is composed of estimating a co-occurrence matrix, $M$, for this abundant set of short tracts. A single element of $M$, namely $M_{i j}$, represents the probability of having the short tracts $S_{i}$ and $S_{j}$ in the same cluster. The MCMC techniques get into play at this stage of SMT.

Let $S_{i}$ be a short tract. Let $\Gamma_{i}$ be a cluster of short tracts that includes $S_{i}$, i.e. a set of short tracts that are on the same fiber. Then, SMT aims at estimating

$$
M_{j i}=P\left(S_{j} \in \Gamma_{i} \mid S_{i} \in \Gamma_{i}\right), M_{i j}=M_{j i}, i, j=1, \cdots, N
$$

where $N$ is the total number of short tracts that populates the complete brain.

Let $S_{i}^{(k)} ; i=1, \cdots, N ; k=1,2$ represent the $k^{t h}$ endpoint of $S_{i}$, without any specific ordering of endpoints. For a short tract $S_{i}$, a bridge is built between $S_{i}^{(1)}$ and the $S_{j}^{(k)}$ with the highest probability of being connected to $S_{i}^{(1)}$. If we denote the position of $S_{i}^{(k)}$ with $r_{i}^{(k)}$ and the diffusion tensor at that position with $D_{i}^{(k)}$, then the probability of bridging $r_{i}^{(1)}$ and $r_{j}^{(k)}$ can be approximated by

$$
\begin{array}{r}
c_{i \rightarrow j}=P\left(r_{i}^{(1)}, r_{j}^{(k)}\right)=\left(\frac{1}{4 \pi \tilde{D}}\right)^{\frac{3}{2}} \exp \left(\frac{-\left\|r_{i}^{(1)}-r_{j}^{(k)}\right\|^{2}}{4 \pi \tilde{D}}\right) \\
\tilde{D}=\frac{\left(r_{i}^{(1)}-r_{j}^{(k)}\right)^{T} D_{i}^{(1)}\left(r_{i}^{(1)}-r_{j}^{(k)}\right)}{\left\|r_{i}^{(1)}-r_{j}^{(k)}\right\|^{2}}
\end{array}
$$

This is the Gaussian distribution as represented by $D_{i}^{(1)}$. Without loss of generality, let all bridges originate from the first end-point $(k=1)$ and terminate in the second end point $(k=2)$. We repeat the whole process starting from $r_{j}^{(1)}$, until no bridge with high enough (an arbitrarily small threshold, $\epsilon$ ) probability can be built. The whole process is repeated starting from the second end-point of $S_{i}$, namely $S_{i}^{(2)}$ (backward clustering). Finally, we get the initial cluster for a given $S_{i}$. Figure 1 depicts bridging from $S_{i}$ to $S_{j}$. 
This initial cluster is a sample from the distribution of all clusters that include $S_{i}$. Let us denote this cluster by $\Gamma_{i}^{0}$. We then increment $M_{i j}$ and $M_{j i}$ by one for all $j$ such that $S_{j} \in \Gamma_{i}^{0}$. The whole process is iterated $K$ times, generating $\left\{\Gamma_{i}^{0}, \ldots, \Gamma_{i}^{K-1}\right\}$. Consecutive iterations are performed by breaking the weakest bridge, building a new one at that location and completing the rest of clustering as explained above. Our goal is to estimate the distribution of such clusters, equivalently, to approximate the probability distribution function (PDF) of the connectivity of $S_{i}$ to all other short tracts. Connectivity between $S_{i}$ and $S_{j}$ is proportional to the probability of the existence of a cluster that includes them both. An approximation to this PDF is the histogram as represented by $M_{i j}, j=$ $\{1, \ldots, N\}$. We used the Metropolis-Hastings algorithm (MHA) to populate the aforementioned histogram [17.

The principal components of MHA are i) a sampling strategy, ii) a sample fitness function, $f($.$) , iii) a candidate generating density, q(.,$.$) , which is the$ probability of generating a new sample from a given sample. Let $\Gamma_{i}^{(m)}$ denote the $m^{\text {th }}$ sample selected from all clusters that include $S_{i}$. The corresponding SMT components are as follows:

1. Sampling Strategy: Given a cluster of short tracts, $\Gamma_{i}^{(m)}$, the weakest bridge is identified. The strength of a bridge between $r_{p}^{(1)}$ and $r_{q}^{(2)}$ is represented by the Fractional Anisotropy (FA) of $D_{p}^{(1)}$ because the reliability of PDD tracking decreases with FA. Let us denote the FA at $D_{p}^{(1)}$ with $F_{p}^{(1)}$. Removing the weakest bridge, the section of $\Gamma_{i}^{(m)}$ that includes $S_{i}$, is retained. A new bridge between $r_{p}^{(1)}$ and one of its neighbours is built at random and a new cluster is formed beyond the new bridge. Let the new bridge be built between $r_{p}^{(1)}$ and $r_{w}^{(2)}$.

2. Sample (Short Tract Cluster) Fitness: The fitness of a sample $\Gamma_{i}^{(m)}$, i.e. $f\left(\Gamma_{i}^{(m)}\right)$ is chosen to be the minimum of the strengths of its bridges because a cluster's reliability is dominated by its weakest bridge.

3. Candidate Generating Density: Probability of generating a new sample candidate cluster from a given one is formulated as the product of the probability of removing the weakest bridge and building a new one. It is given as,

$$
q\left(\Gamma_{i}^{(m)}, \Gamma_{i}^{(m+1)}\right)=\underbrace{\frac{1 / F_{p}^{(1)}}{\sum_{j \in A} 1 / F_{j}^{(1)}}}_{\text {Prob. of removing a bridge }} \times \underbrace{\frac{c_{p \rightarrow w}}{\sum_{z \in B} c_{p \rightarrow z}}}_{\text {Prob. of building a bridge }}
$$

where $F_{p}^{(1)}$ is the fitness of the removed bridge, $c_{p \rightarrow w}$ is the probability of the newly built bridge originating from $r_{p}^{(1)}, A$ is the set of short tract indices that belong to $\Gamma_{i}^{(m)}$ and $B$ is the set of short tract indices that are in the neighbourhood of $r_{p}^{(1)}$. 
For a given seed tract $S_{i}$, the MHA is iterated. The newly generated sample at each iteration is accepted with a probability given as,

$$
\alpha\left(\Gamma_{i}^{(m)}, \Gamma_{i}^{(m+1)}\right)=\min \left(1, \frac{\left.f\left(\Gamma_{i}^{(m+1)}\right) q\left(\Gamma_{i}^{(m)}, \Gamma_{i}^{(m+1)}\right)\right)}{f\left(\Gamma_{i}^{(m)}\right) q\left(\Gamma_{i}^{(m+1)}, \Gamma_{i}^{(m)}\right)}\right)
$$

If $\Gamma_{i}^{(m+1)}$ is accepted, then we increment $M_{i n}, M_{n i} \forall S_{n} \in \Gamma_{i}^{(m+1)}$, otherwise, we increment $M_{i n}, M_{n i} \forall S_{n} \in \Gamma_{i}^{(m)}$ by one. The number is iterations, $K$, is empirically determined to be 100 . The whole process repeated to build $M$ by taking each short tract as the seed tract.

The co-occurrence matrix $M$ is computed and saved off-line. It represents the whole brain connectivity. The user is required to select a volume of interest to mark a set of seed tracts and a confidence threshold, $\tau$. For each seed tract $S_{i}$ in the volume interest, all $S_{j}$ 's with $M_{i j} \geq \tau \times K$ are selected and displayed. The interface is similar to the dynamic queries interface proposed in [18].

\section{Results}

We used real patient DT-MRI data for the initial validation of the SMT method. The scans were single-shot EPI scans with diffusion encoding along 12 noncollinear directions plus one reference without diffusion-weighting. The FOV was $25-26 \mathrm{~cm}$, TE was minimum with partial k-space acquisition. TR was $\sim 10 \mathrm{~s}$ and b-value was $\sim 850 \mathrm{~s} / \mathrm{mm}^{2}$.

Seed tracts are selected with a spherical volume of interest (VOI) on the left side of the corpus callosum / optic radiation of a healthy individual, as marked with circles in Figures $2 a a$ and $2 b$. Seed volumes are identical for both images. The confidence threshold, $\tau$, for Figures $2 a$ and $b$ are 0.0 and 0.1 , respectively. Note the decrease of the number of short tracts with increasing confidence.

A second set of seed tracts are selected with a spherical VOI at the inferior part of the cortico-spinal tracts of the same healthy individual as shown in Figures 2 $c$ and $2 d$. The VOI covers both the left and the right sides. The cortico-spinal tracts are known to spread as they extend to the superior regions. The confidence threshold, $\tau$, for Figures $2 c$ and $2 d$ are 0.0 and 0.3 , respectively. In addition to the effect of $\tau$, we can also observe the branching that SMT allows.

The final set of seed tracts are selected with a spherical VOI in the inferior longitudinal fasciculus region, close to the uncinate fasciculus, as marked with circles in Figures $2 e$ and $2 f$. The confidence threshold, $\tau$, is 0.0 and 0.6 , respectively.

Computation of the short tracts (13540 short tracts for the current dataset) throughout the brain and the co-occurrence matrix $M$ takes approximately 3 hours on $\mathrm{PC}$ with Pentium $4(2.4 G H z)$ and $1.5 G B$ RAM. This computation is performed once for each dataset in batch mode and $M$ is saved. Visualization and analysis of the data based on the computed $M$ is a real-time application. 


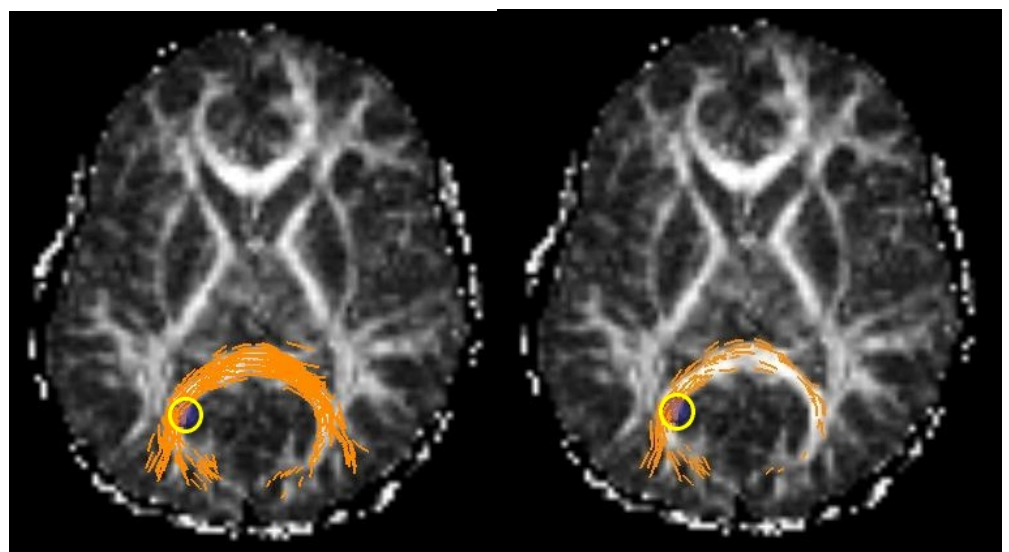

(a)

(b)

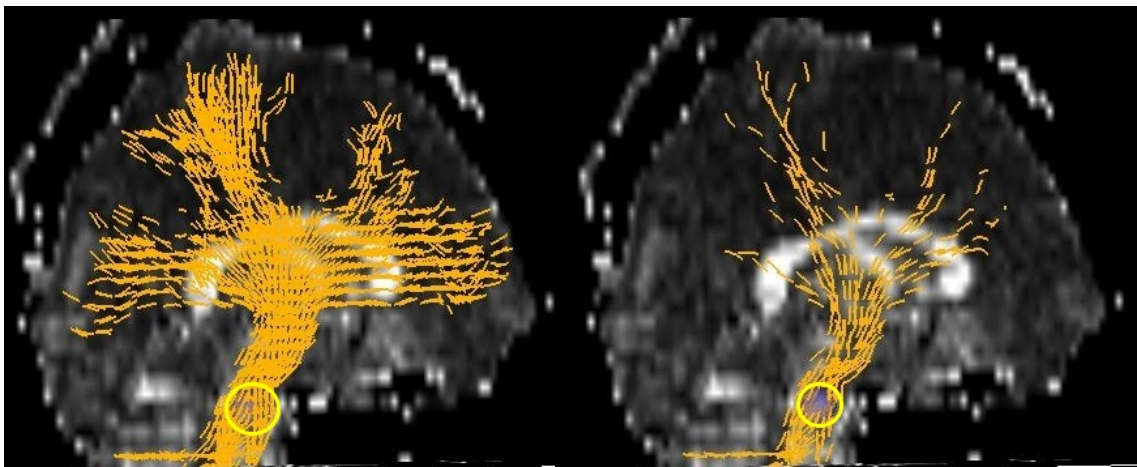

(c)

(d)

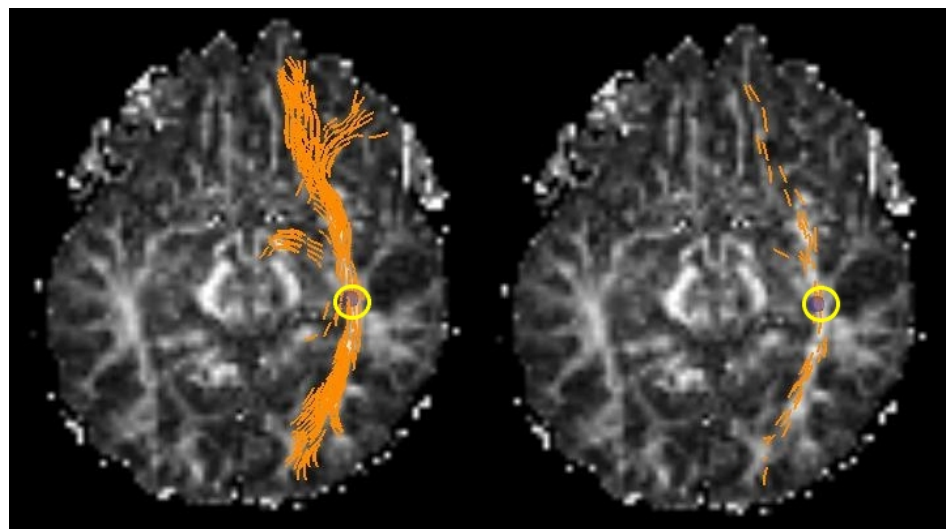

(e)

(f)

Fig. 2. Three sets of seed tracts, one for each row, in different regions of the brain of a healthy human are selected and the corresponding short tract clusters with different confidence levels (low for the left images, high for the right images) are displayed 


\section{Discussion}

The SMT method combines the intuitive interpretation of conventional fiber tractography with the stochastic approach of connectivity analysis using a Markov Chain Monte Carlo (MCMC) framework. It is based on estimating the PDF of the cluster of short tracts connected to a given a seed tract using the Metropolis-Hastings algorithm [17.

The advantage of using short tracts is the intuitive user interface that it provides and the negligible tracking error accumulation. SMT displays all short tracts connected to a given seed tract with a probability higher than the user set confidence threshold. The interpolation of the complete tracts is left to the human visual system. The efficiency of this interpolation increases with the increasing density of short tracts. This approach provides a direct way to present the information content of DT-MRI data by explicitly displaying the possibilities at problematic regions, such as kissing and crossing fibers.

The MCMC framework, on the other hand, exploits the stochastic nature of DT-MRI data. The data is based on the second order approximation of the total diffusion of water molecules within a finite subvolume (the voxel) in a given direction (as determined by the diffusion weighting gradient fields), observed through an attenuation in MR signal received. In other words, the computed diffusion tensors represent the probabilistic spatial distribution of diffusing molecules in a given voxel within a given time period. Consequently, it is more accurate to consider this distribution, as done in connectivity analysis, than to consider the principal diffusion direction only, as mostly done in conventional fiber tractography. The Metropolis-Hastings algorithm utilizes this information in estimating the clusters of short tracts. It, thus, allows for branching, merging and crossing pathways.

Although the computational cost of computing the co-occurrence matrix $(M)$ is high, it is performed once for each dataset, in batch mode and independent of any VOI. A single $M$ matrix describes the connectivity throughout the brain. We have used 100 iterations of the Metropolis-Hastings algorithm. Increasing the number of iterations would increase the accuracy, yet we have not observed significant differences in the results when the number of iterations is increased beyond 100. The examination of DT-MRI data is based on dynamic queries that define VOIs and is real-time [18.

The SMT method proposes a framework based on clustering short fiber tracts with Markov Chain Monte Carlo techniques, specifically with the MetropolisHastings Algorithm. We have presented the underlying model and preliminary results. Neither the proposed sampling strategy nor the sample fitness or the candidate generating density is claimed to be the optimum choices. Different tractography methods can be developed within the SMT framework simply by using different models for these components. Research on variations of SMT, its performance with high b-value data and a thorough clinical evaluation is left as future work.

\section{Acknowledgments}

This work was in part supported by TÜBITTAK KARIYER-DRESS (104E035) project, Boğaziçi University B.A.P. DTIsuite (07A203) project and EU $6^{\text {th }} \mathrm{FP}$ 
SIMILAR NoE. We thank to Prof. Roland Bammer from Stanford University for his comments and providing the DT-MRI data.

\section{References}

1. Basser, P., Mattiello, J., LeBihan, D.: Estimation of the effective self-diffusion tensor from the NMR spin echo. J. Magn. Reson. B 103, 247-254 (1994)

2. Basser, P.: Inferring microstructural features and the physiological state of tissues from diffusion-weighted images. NMR Biomed. 8, 333-344 (1995)

3. Basser, P., Pierpaoli, C.: Microstructural and physiological features of tissues elucidated by quantitative diffusion tensor mri. J. Magn. Reson. B 111, 209-219 (1996)

4. Liu, C., Bammer, R., Acar, B., Moseley, M.: Characterizing non-gaussian diffusion by using generalized diffusion tensors. Magnetic Resonance in Medicine 51, 924-937 (2004)

5. Basser, P., Pajevic, S., Pierpaoli, C., Duda, J., Aldroubi, A.: In vivo fiber tractography using dt-mri data. Magn. Reson. Med. 44, 625-632 (2000)

6. Tench, C., Morgan, P., Wilson, M., Blumhardt, L.: White matter mapping using diffusion tensor MRI. Magnetic Resonance in Medicine 47, 967-972 (2002)

7. Bammer, R., Acar, B., Moseley, M.: In vivo mr tractography using diffusion imaging. European J. Radiology 45, 223-234 (2003)

8. Lazar, M., Alexander, A.: An error analysis of white matter tractography methods: synthetic diffusion tensor field simulations. Neuroimage 20, 1140-1153 (2003)

9. Koch, M., Norris, D., Hund-Georgiadis, M.: An investigation of functional and anatomical connectivity using magnetic resonance imaging. Neuroimage 16, 241250 (2002)

10. Hagmann, P., Thiran, J., Vandergheynst, P., Clarke, S., Meuli, R.: Statistical fiber tracking on DT-MRI data as a potential tool for morphological brain studies. In: ISMRM Workshop on Diffusion MRI: Biophysical Issues (2000)

11. Chung, M., Lazar, M., Alexander, A., Lu, Y., Davidson, R.: Probabilistic connectivity measure in diffusion tensor imaging via anisotropic kernel smoothing. Technical Report 1081, University of Wisconsin (2003)

12. Lenglet, C., Deriche, R., Faugeras, O.: Diffusion tensor magnetic resonance imaging: Brain connectivity mapping. Technical Report 4983, INRIA, Sophia-Antipolis, France (2003)

13. Bozkaya, U.: SMT: Split/merge fiber tractography for MR-DTI. Master's thesis, Bogazici University, Biomedical Engineering Institute, Istanbul, Turkey (2006)

14. Bozkaya, U., Acar, B.: SMT: Split/merge fiber tractography for MR-DTI. In: ESMRMB 2006, Warsaw, Poland (2006)

15. Hagmann, P., Thiran, J., Jonasson, L., Vandergheynst, P., Clarke, S., Maeder, P., Meuli, R.: DTI mapping of human brain connectivity: statistical fibre tracking and virtual dissection. Neuroimage 19, 545-554 (2003)

16. Lazar, M., Alexander, A.: Bootstrap white matter tractography (BOOT-TRAC). Neuroimage 24, 524-532 (2005)

17. Chib, S., Greenberg, E.: Understanding the Metropolis-Hastings algorithm. The American Statistician 49, 327-335 (1995)

18. Sherbondy, A., Akers, D., Mackenzie, R., Dougherty, R., Wandell, B.: Exploring connectivity of the brain's white matter with dynamic queries. IEEE Trans. Vis. Comput. Graph. 11, 419-430 (2005) 